\title{
The Effect of Gas Atmospheres on Charging
}

*Xiaohu Tang and ${ }^{* *}$ David C. Joy

*Electron Microscope Facility, University of Tennessee, Knoxville, TN 37996-0810 USA

** Oak Ridge National Laboratory, Oak Ridge, TN 37831-6064 USA

Charging accumulation in insulating or semiconducting samples due to electron beam irradiation is one of the most important problems in electron microscopy. One of the most promising techniques for reducing the severity of such charging is to surround the sample with a low-pressure atmosphere of a gas ${ }^{[1]}$. Although the earliest workers reported that charging is eliminated in the presence of gas, it is now clear that in general charging is only stabilized and reduced in this way. We have therefore measured the charging behavior of a number of materials, and several different gases, to determine the important factors in charge control under these conditions. Charging was determined by measuring the Duane-Hunt (D-H) cut-off of the X-ray spectrum from the selected sample.

The X-ray energy-dispersive-spectra (EDS) were obtained using a Gresham X-ray detector installed on a Hitachi S-3500 SEM under VP mode. All experiments were performed using mica, mineral (Fe, $\mathrm{Mg}, \mathrm{Si}$ and $\mathrm{O}$ ) and sapphire specimens. At a given electron beam energy and zero pressure, the sample current was adjusted to $0.1 \mathrm{nA}$ by adjusting the condenser lens in order to set a standard for comparison. The gas pressure was varied from $270 \mathrm{~Pa}$, then down to $200 \mathrm{~Pa}, 100 \mathrm{~Pa}, 30 \mathrm{~Pa}, 10 \mathrm{~Pa}, 3 \mathrm{~Pa}$ and finally to $1 \mathrm{~Pa}$ so as to minimize the residual ionization from previous runs. The above procedure was repeated at $10 \mathrm{keV}, 15 \mathrm{keV}, 20 \mathrm{keV}, 25 \mathrm{keV}$ and $30 \mathrm{keV}$ beam energy. Finally these experiments were repeated using an atmosphere of helium rather than air. The D-H limit was determined from the routine in DTSA which performs a linear regression on the top 100 channels of the spectrum ${ }^{[2]}$. The magnitude of the surface charging was then determined by subtracting the measured D-H cut-off value from the nominal incident beam energy ${ }^{[3]}$. If the sample is charging strongly then electrons can be re-accelerated from the surface and be scattered in the column leading to a false or ambiguous D$\mathrm{H}$ limit.

The D-H value obtained from the X-ray spectrum was converted into the surface charging. Figure 1 shows the experimentally determined variation of surface charging, plotted in units of kilo-electron volts, as a function of gas pressure for air over the range of $1 \mathrm{~Pa}$ to $270 \mathrm{~Pa}$ on the surface of sapphire. The surface charging decreases quickly as the air pressure is increased then reaches to a stable state. There exists a charge balance point for $10 \mathrm{keV}$ electrons but as the beam energy is increased, the sample holds a negative but stable surface charge and does not reach the charge balance. At the lower gas pressures $(<20 \mathrm{~Pa})$, surface charging has an approximately linear relationship with the logarithm of the air pressure. After replacing the air atmosphere by helium, the relationship of surface charging and gas pressure is shown in Figure 2. Both gas atmospheres have a similar trend except for differences in the value of stable surface charging, charge balance point and charging reduction efficiency (CRE). CRE is the slope of the profile $\square \mathrm{V}$ vs $\square$ Pressure. In the helium atmosphere, the gas pressure corresponding to the charge balance is higher than that in air atmosphere. This implies that air is more efficient in removing charge than helium. Comparable phenomena have been observed for other materials and gases.

\section{References}


[1] Adamiak B and Mathieu C, Scanning vol. 22, 178-181 (2000).

[2] Reed SJB, Electron Microprobe Analysis, $2^{\text {nd }}$ edition. Cambridge University Press (1993).

[3] Newbury DE, Scanning vol. 22, 345-351 (2000).

[4] This work was supported by SRC contract LJ-413.003. The contract monitor is Dr. D. Herr.

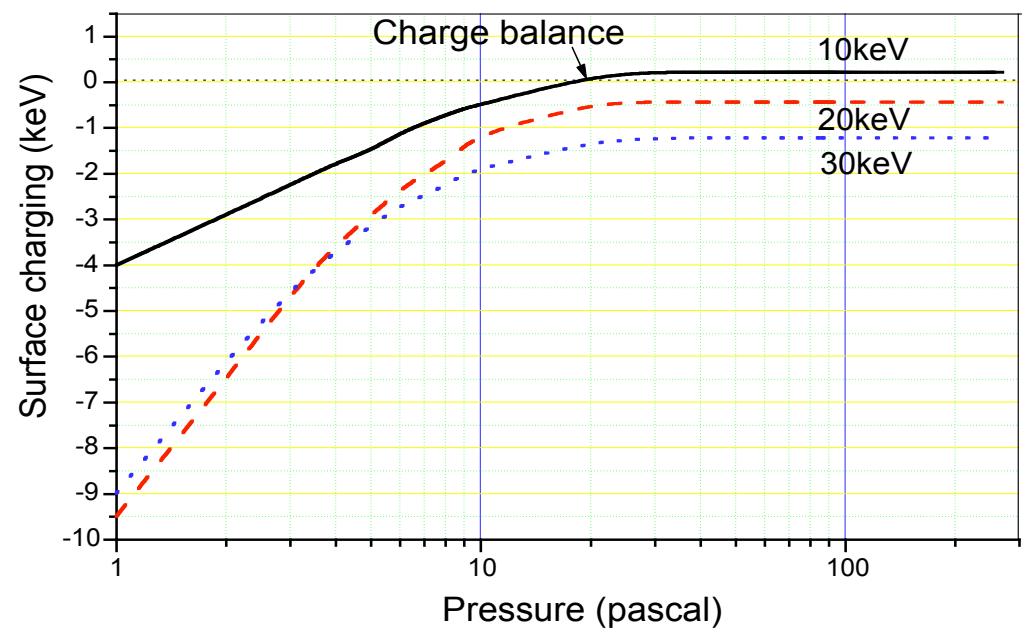

FIG. 1. The surface charging as a function of air pressure for electron beam energy as 10,20 and $30 \mathrm{keV}$

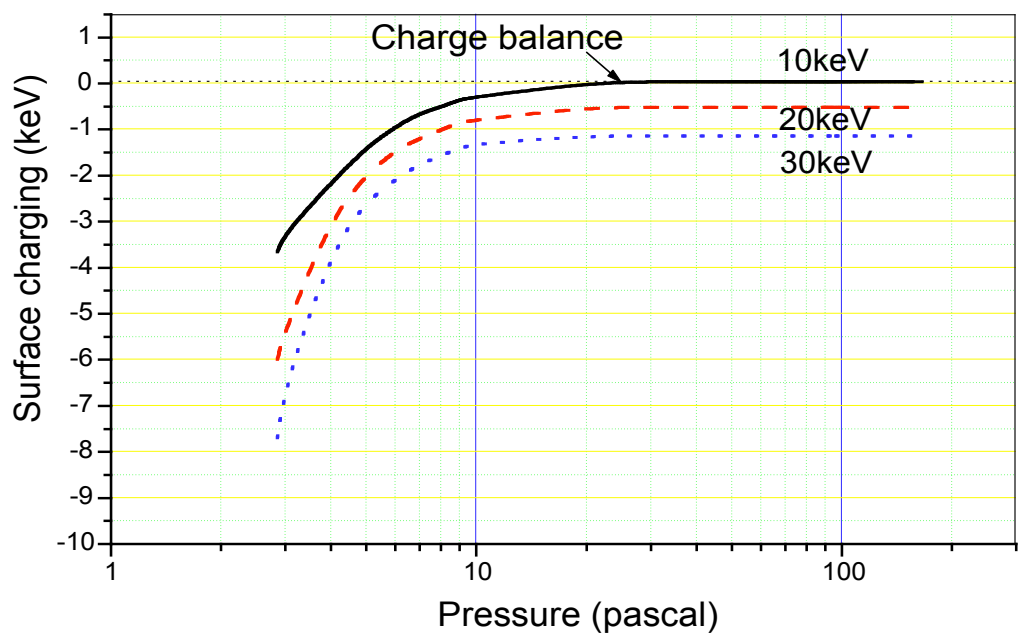

FIG. 2. The surface charging as a function of helium pressure for electron beam energy as 10,20 and $30 \mathrm{keV}$ 